\title{
Assessment of the mixing efficiency of solid mixtures by means of image analysis
}

\author{
B. Daumann *, H. Nirschl \\ University of Karlsruhe (TH), Institute for Mechanical Process Engineering and Mechanics, D-76128 Karlsruhe, Germany
}

Received 7 November 2006; received in revised form 4 June 2007; accepted 5 July 2007

Available online 13 July 2007

\begin{abstract}
When assessing a mixing process, mixing quality is a characteristic parameter. To determine the optimum mixing time, it is necessary to measure the mixing efficiency as a function of mixing time. Mixing efficiency is determined by a sufficient number of sample analyses after certain mixing times. The novel method of image analysis allows to rapidly determine the optimum mixing time without sampling and complex sample analysis being required. In this study the model products have different particle sizes and colors to see a difference between them in the image analysis program. Analyzing a real mixture to find chemical substitutes for all particle components is impossible. The study can help the plant engineer to mark a component of interest for finding the optimum point of stationary equilibrium. In this paper the theory for sampling and comparing multi-component mixtures by image analysis to determine the mixing efficiency will be also described and discussed.
\end{abstract}

(C) 2007 Elsevier B.V. All rights reserved.

Keywords: Solid mixing; Mixing efficiency; Image analysis; Multi-component mixture

\section{Introduction}

To assess the homogeneity of solid mixtures and to determine the necessary mixing time and distribution of the solid concentration in the mixing volume, a sufficient number of samples must be taken. Outside of the mixer, these samples have to be subjected to relatively complex analyses. Sampling always represents an irreversible intervention at the sampling point, which significantly influences the surrounding mixture. However, systematic sampling at defined points is necessary for comparisons of mixtures under various boundary conditions and initial states. Detailed discussions can be found in Sommer and Hauser [1] and Raasch and Elsässer [2].

Depending on the statistical requirements, the sample size is about $4 \mathrm{~g}$ to $50 \mathrm{~g}$ in case of $\mu \mathrm{m}$ particles. Using particles of several millimeters, the sample mass needed to make an exact statistical statement on the basis of the measurements will amount to several hundred grams or even kilograms. Consequently, the methods presently applied to determine the mixing

\footnotetext{
* Corresponding author. Tel.: +49 721608 4139; fax: +49 7216082403.

E-mail address: bjoern.daumann@mvm.uka.de (B. Daumann).
}

efficiency of millimeter particles are characterized by the mixer having to be discharged completely. As discharge inevitably leads to demixing, no conclusions can be drawn with respect to the mixing efficiency in the mixer.

The studies described below were carried out using a $60 \mathrm{~L}$ single-shaft mixer made by the Elba company, Germany. In practice the mixer is used to produce dry mixtures, mortars and concretes of variable quality. Work focused on the development of a method that documents the mixing efficiency by digital imaging of the surface rather than by sampling. Then, mixing efficiency is assessed by image analysis. The mixing qualities obtained from both methods, namely image analysis and sieving, will then be compared.

\section{Theoretical considerations}

\subsection{Fundamentals of the determination of mixing quality}

The mixing quality $\sigma^{2}$ reflects the homogeneity of a mixture and reveals the extent to which mixing of the individual components of the solid mixture deviates from the required value. Fig. 1a and b illustrate potential mixing states of a threecomponent mixture. 


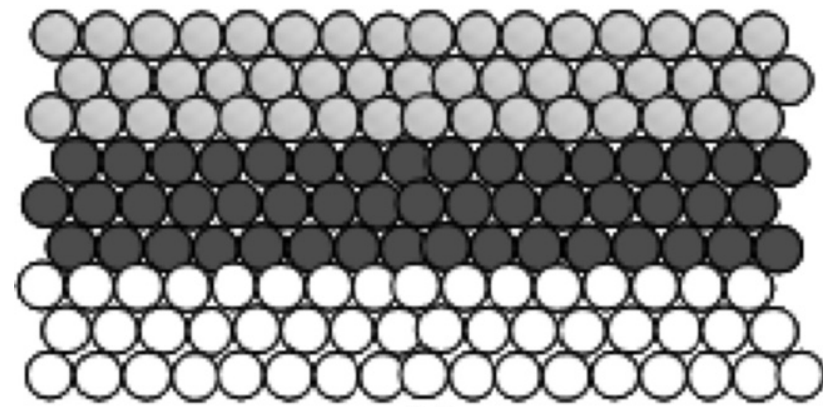

(a)

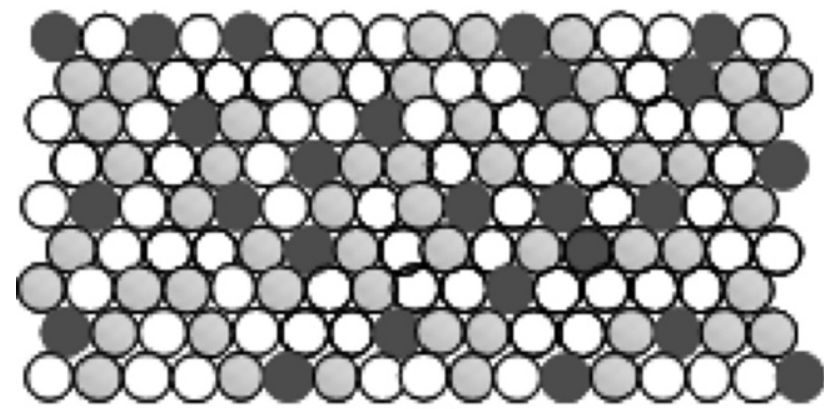

(b)

Fig. 1. a) Complete demixing $\sigma^{2}{ }_{0}$, b) ideal disorder $\sigma^{2}{ }_{z}$.

To calculate the individual variances $\sigma^{2}$ of the mixing states, the following equation is applied.

$\sigma^{2}\left(X_{j}\right)=\lim _{N \rightarrow \infty} \frac{1}{N} \cdot \sum_{i=1}^{N}\left(X_{j, i}-P_{j}\right)^{2}$

Eq. (1) yields the quadratic deviation from the target value $P_{j}$ for the sample concentration $X_{j}$. From several samples $N$ of the fraction of the component $j$, the variance $\sigma^{2}$ is obtained. In case the number of samples $N$ does not approach infinity, the variance $\sigma^{2}$ becomes the empirical variance $s^{2}$, see Eq. (2).

$s^{2}\left(X_{j}\right)=\frac{1}{N} \cdot \sum_{i=1}^{N}\left(X_{j, i}-P_{j}\right)^{2}$

According to Eq. (3.1), the variation coefficient $v$ is obtained from the standard deviation $s$ and the target value $P_{j}$. From Eq. (3.2) follow that, this variation coefficient reflects the percentage deviation from the target value $P_{j}$.

$v=\frac{s}{P_{j}}$

$X_{j}=P_{j} \pm v$

The completely demixed state of a three-component mixture (Fig. 1a) is calculated using Eq. (4).

$\sigma_{0}^{2}=P_{j} \cdot\left(1-P_{j}\right)$

As pointed out by Sommer [3], the variance of ideal disorder $\sigma_{Z}^{2}$ of a multi-component mixture (Fig. $1 \mathrm{~b}$ ) at a constant sample mass $m_{P}$ or a ratio of the particle fractions of $x_{j} / x_{j+1} \approx 1.26$ can be calculated by Eqs. (5.1)-(5.3). Eqs. (5.1) and (5.2) are influenced by the individual grain size $m_{E, j}$, the sample mass $m_{P}$, and the mass fraction $P_{j}$. Eq. (5.1) gives the ideal disorder $\sigma_{Z}^{2}$ of the individual component. The equation consists of the addition of two terms. The first term of Eq. (5.1) is the stochastic homogeneity $\sigma_{Z}^{2}$ of the component $j$ of the mixture to be studied. The second term of Eq. (5.1) denotes the calculation of stochastic homogeneity $\sigma_{Z}^{2}$ of the particle size larger than the component $j$. The variance $\sigma_{Z}^{2}\left(\Sigma X_{i}\right)$ is calculated by means of Eq. (5.2). This equation gives the total of all stochastic variances for the mixture. The value of the variance for $j=1$ according to Eq. (5.2) is the same than in Eq. (5.1). For the last component $j$, the variance in Eq. (5.2) is $\sigma_{Z}^{2}\left(\Sigma X_{i}\right)=0$. Eq. (5.3) calculates the value expected $P_{E}$. This value gives the ratio between the mass fraction of the component $j$ and the residual fraction of the total mixture. Assuming that the influence of the particle ratio on the material selected does not influence the calculation of stochastic homogeneity, the following equations are valid.

$\sigma_{Z}^{2}\left(X_{j}\right)=P_{E} \cdot\left(1-P_{E}\right) \cdot \frac{m_{E, j}}{m_{P}} \cdot\left(1-\sum_{i=1}^{j-1} P_{i}\right)+P_{E}^{2} \cdot \sigma_{Z}^{2}\left(\sum_{i=1}^{j-1} X_{i}\right)$

$\sigma_{Z}^{2}\left(\sum_{i=1}^{j} X_{i}\right)=P_{K} \cdot\left(1-P_{E}\right) \cdot \frac{m_{E, j}}{m_{P}}+\left(1-P_{E}\right)^{2} \cdot \sigma_{Z}^{2}\left(\sum_{i=1}^{j-1} X_{i}\right)$

$P_{E}=\frac{P_{j}}{1-\sum_{i=1}^{j-1} P_{i}}$

The mixing efficiency obtained from sieving $\sigma_{S}^{2}\left(X_{j}\right)$ according to Eq. (6) is time-dependent and consists of three variances, see Sommer [3]. The variance of the measurement method $\sigma_{M}^{2}$ denotes the reproducibility and is obtained from preliminary tests. Usually, it should be small, such that the variance of the random mixture $\sigma_{Z}^{2} \gg \sigma^{2}{ }_{M}$ only influences the system and the variance of the measurement method can be neglected. Systematic variance $\sigma_{\text {syst. }}^{2}$ is time-dependent and has the value of $\sigma_{\text {syst. }}^{2}=0$ in the stationary state, i.e. the mixing process is completed. A longer mixing time does not improve the mixing quality. The variance $\sigma_{S}^{2}\left(X_{j}\right)$ according to Eq. (6) is calculated by adding the quadratic deviations from the target value $P_{j}$ of the individual samples $N$. By determining the variance at different mixing times, the mixing efficiency is obtained.

$\sigma_{S}^{2}\left(X_{j}\right)=\sigma_{Z}^{2}+\sigma_{M}^{2}+\left(1-\frac{m_{E, j}}{m_{P}}\right) \cdot \sigma_{\text {syst. }}^{2}\left(t_{M}\right)=\frac{1}{N} \cdot \sum_{i=1}^{N}\left(X_{j, i}-P_{j}\right)^{2}$

The sample mass $m_{P}$ influences the variance according to Eqs. (5.1) and (6). Eq. (7) of Alex [4] is used to determine the 
Table 1

Material characterization

\begin{tabular}{llll}
\hline Material data & Coarse fraction & Medium-sized fraction & Fine fraction \\
\hline Solid density $\rho_{s}$ & $2700 \mathrm{~kg} / \mathrm{m}^{3}$ & $2700 \mathrm{~kg} / \mathrm{m}^{3}$ & $2740 \mathrm{~kg} / \mathrm{m}^{3}$ \\
Bed density $\rho_{\text {bed }}$ & $1400 \mathrm{~kg} / \mathrm{m}^{3}$ & $1400 \mathrm{~kg} / \mathrm{m}^{3}$ & $1430 \mathrm{~kg} / \mathrm{m}^{3}$ \\
Residual humidity $x_{R}$ & $<0.1 \%$ & $<0.1 \%$ & $<0.1 \%$ \\
Particle size $x_{V}$ & $13.7 \mathrm{~mm}$ & $5.3 \mathrm{~mm}$ & $0.7 \mathrm{~mm}$ \\
Single particle mass & $2.5 \mathrm{~g}$ & $0.42 \mathrm{~g}$ & $0.001 \mathrm{~g}$ \\
Sphericity $\psi$ & 0.76 & 0.67 & 0.74 \\
\hline
\end{tabular}

minimum sample mass for a single sample. If the individual sample size is normally distributed, the following relationship applies according to Alex.

$m_{P} \geq\left(t_{s} / v\right)^{2} \cdot\left(1-P_{j}\right) \cdot P_{j} \cdot m_{E, j}$

In Eq. (7), $m_{P}$ is the sample mass, $P_{j}$ the mass fraction, and $j$ the main component. The student factor $t_{s}$ and the relative deviation $v$ from the target value are given or may be chosen. Other influencing parameters in the equation are the single grain weight $m_{E}$. Calculating Eq. (7) with $t_{S}=1.91, v=0.07, P_{j}=0.33$ and $m_{E, 1}=2.5 \mathrm{~g}$ means, that the maximum particle size dominates the minimum sample mass, which contains between $400 \mathrm{~g}$ and $600 \mathrm{~g}$ of product.

\subsection{Fundamentals of the determination of mixing quality by image analysis}

The variance of image analysis $\sigma_{B}^{2}\left(X_{j}\right)$ can be determined using Eq. (8). The difference between Eqs. (8) and (6) is that the surface fractions are determined rather than the mass fractions in the individual sample. The measurement error $\sigma_{M}^{2}$ is assumed to be $\sigma_{M}^{2}=0$. The variance $\sigma_{B}^{2}\left(X_{j}\right)$ is obtained from the quadratic deviation from the target value of the individual digital images.

$$
\begin{aligned}
\sigma_{B}^{2}\left(X_{j}\right) & =\sigma_{Z}^{2}+\sigma_{M}^{2}+\left(1-\frac{m_{E, j}}{m_{P}}\right) \cdot \sigma_{\text {syst. }}^{2}\left(t_{M}\right) \\
& =\frac{1}{N} \cdot \sum_{i=1}^{N}\left(\frac{A_{j}}{A_{\text {ges }}}-P_{j}\right)^{2}
\end{aligned}
$$

To determine the stochastic variance $\sigma_{Z}^{2}$ for image analysis, the sample mass at the surface of the mixing volume must be known. The total mass $m_{P}$ of the sample can be calculated using Eqs. (9.1)-(9.4).

Following the definition of Wadell [5], sphericity $\psi$ is obtained from the ratio between the surface of the equal volume sphere and the real particle surface using Eq. (9.1). When applying the Cauchy's theorem, the mean projected surface of the individual particle exceeds the real particle surface by a factor of four. The individual grain weight $m_{E, j}$ of a sphere may be determined by Eq. (9.2). The particle number of the individual components is described by Eq. (9.3). The total area $A_{\text {ges }}$. in Eqs. (9.3) and (9.4) is given by the image evaluation program. The sphericity and equal volume diameter $x_{V}$ may be taken from Table 1, the target value $P_{j}$ from the preceding weighting of the individual fractions. The sample mass $m_{P}$

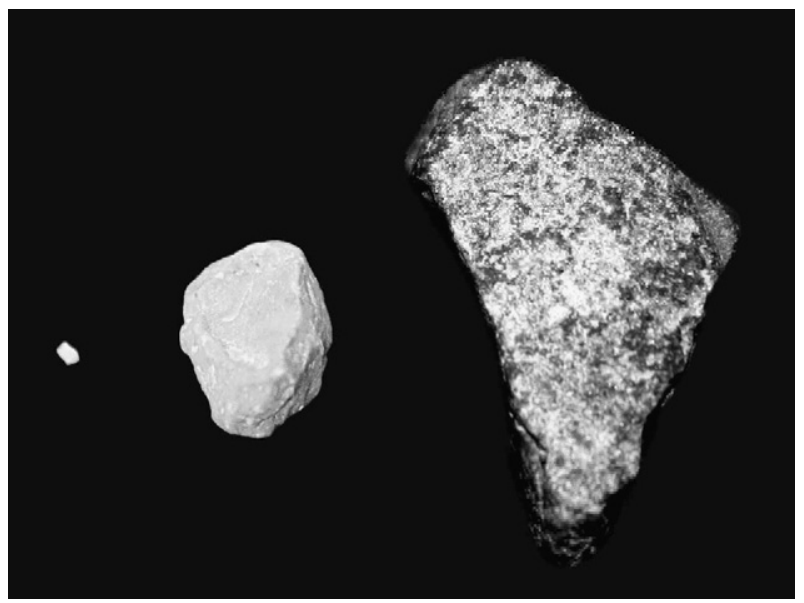

Fig. 2. Size differences of the particle fractions.

calculated according to Eq. (9.4) allows to determine the stochastic homogeneity $\sigma_{Z}^{2}$ for image analysis according to Eqs. (5.1)-(5.3).

$$
\begin{aligned}
& \psi=\left(\frac{x_{V}}{x_{\mathrm{pm}}}\right)^{2}=\left(\frac{x_{V}}{x_{\mathrm{pm}}}\right)^{2} x_{S}=x_{\mathrm{pm}} \Rightarrow \text { Cauchy } \\
& m_{E, j}=\rho_{S} \cdot \frac{1}{6} \cdot \pi \cdot x_{V}^{3} \\
& N_{E, j}=4 \cdot \frac{A_{\mathrm{ges} .} \cdot P_{j}}{x_{\mathrm{pm}}^{2} \cdot \pi} \\
& m_{P}=\sum_{j=1}^{N} m_{E, j} \cdot N_{E, j}=\frac{2}{3} \cdot \rho_{P} \cdot A_{\mathrm{ges}} \cdot \sum_{j=1}^{N} \cdot P_{j} \cdot x_{V} \cdot \psi
\end{aligned}
$$

By using these equations to calculate the total mass of the surface layer of the solid mixer, the resulting whole mass is approximately $m_{P} \approx 600 \mathrm{~g}$. An arrangement of sieving operation

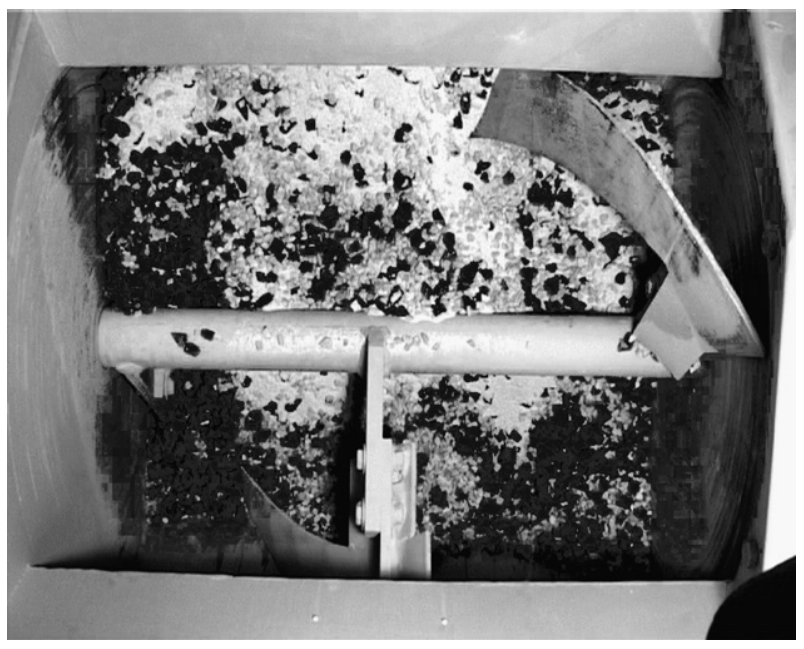

Fig. 3. Distribution of the particles in the mixing volume after $20 \mathrm{~s}$. 


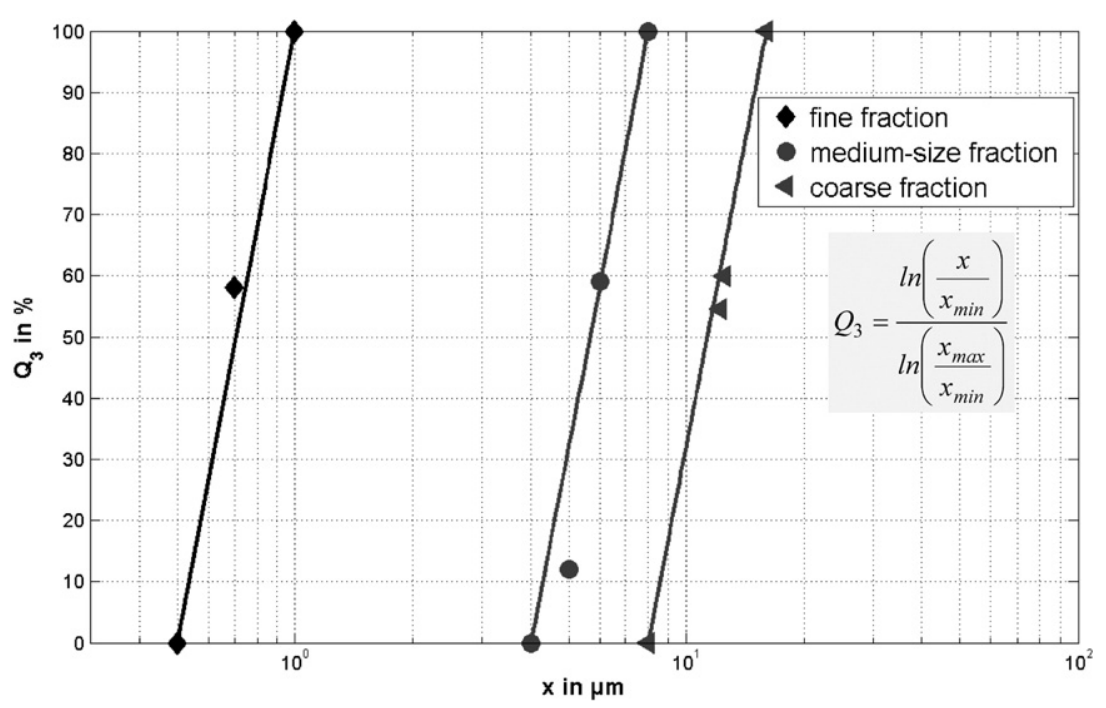

Fig. 4. Particle size distribution of the feeding material.

and image analysis is possible, if the total mass is almost the same.

\section{Preparation of image analysis and material characterization}

Images made at the surface of a mixer can only be analyzed when a digital video camera Sony VX 2100E can access and light sources with thousand watt are available. Shadows due to the mixing tool and the trough edge must be prevented by illumination and optimum positioning of the camera. For the studies presented here, special colored particles were used, which can be distinguished clearly in the mixing volume. It was demonstrated by preliminary tests that the materials (Fig. 2)) can be distinguished by the image evaluation program. Each individual digital image is a copy of a certain mixing state in the mixing volume at a certain time (Fig. 3). A sufficient particle size in the range of several millimeters is advantageous for this type of image analysis, as size differences/ color differences are clearly visible. The colored particle doesn't have the same color like the varnishing of the solid mixer.

For the studies, three fractions were selected, which differed in particle size, but not in solid density $\rho_{s}$ and only slightly in sphericity $\psi$. The parameters measured are listed in Table 1 . The particle size distribution (Fig. 4) of the individual fractions was chosen to vary in a very narrow range. However, overlapping distributions were to be avoided for a later analytical sieving in comparison with image analysis.

\section{Studies in the single-shaft mixer}

\subsection{Description of the mixer}

The tests were carried out using a horizontal single-shaft mixer made by the company of Elba Baumaschinen $\mathrm{GmbH}$, Germany. This mixer type consists of a spiral mixing tool. The mixing tool with a diameter of $D_{\mathrm{W}}=550 \mathrm{~mm}$ shown in Fig. 8 is installed in a horizontal cylindrical mixing tank. The diameter of the mixing tank is $D=560 \mathrm{~mm}$. The ratio between the diameter and length of the mixing tank is approximately $D / L \approx 0.93$. The optimum of utilization level $\varphi$ is between $45 \%$ and $50 \%$ and the visible surface without mixing equipment, covered with product is approximately $A=0.09 \mathrm{~m}^{2}$.

\subsection{Calculation of the threshold by using image analysis}

Fig. 5 shows the procedure of digital images for separating the three different particle fractions. The first step is to create a cavity for the mixer wall and visible part of the shaft. After
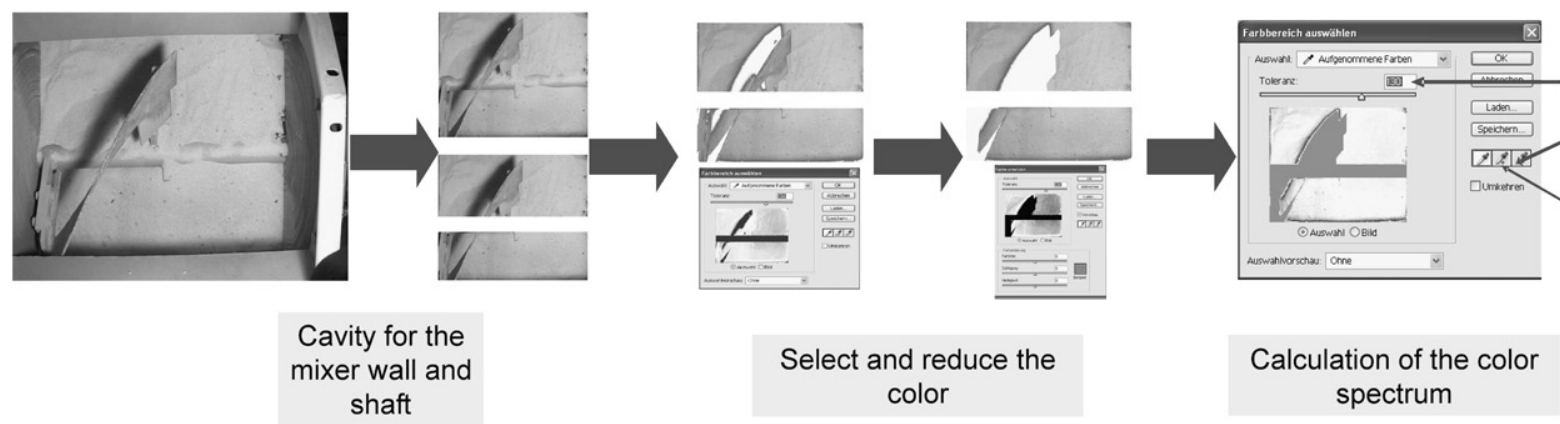

tolerance

reduce color to color selection

add color to color selection

Cavity for the shaft
Select and reduce the color
Calculation of the color spectrum

Fig. 5. Steps to get the threshold for the particle fractions. 


\begin{tabular}{|c|c|c|}
\hline fine fraction & medium-size & coarse fraction \\
\hline coarse fraction & fine fraction & medium-size \\
\hline medium-size & coarse fraction & fine fraction \\
\hline
\end{tabular}

Fig. 6. Potential demixing states.

cutting the digital images, the fractions will be selected and the color will be reduced, because the position of the mixing paddles change during the experiments. To use image analysis, a threshold has to be defined. The method doesn't work if the operating conditions change during the exposure. A strong influence on the image analysis comes from the light intensity and the camera position. Effects like shadow or reflection of the particles can falsify the results. The image analysis is based on different colors of particles. In preliminary tests only one particle fraction, like the fine fraction in Fig. 5, is present in the mixing tank. The preprocessing of the digital images to calculate the threshold is performed with the image processing code "Adobe Photoshop 7.0". The code has to identify the colour spectrum for the fine-, medium- and coarse fraction. If the conditions are stable, the colour white in Fig. 5 does not change the intensity. By the application of the Adobe Photoshop "colour replace" it is possible to define a threshold between the three colors. A macro will be created in Adobe Photoshop to save the settings, which doesn't change during the study. The calculation settings can be used in the three-component mixture during a mixing process. The code separates all three particle fractions into three separate images.

\subsection{Execution of the experiments}

As evident from Fig. 6, the dry components are fed into the mixer from the lower trough bottom up to the surface along the mixing tool axis. The initial state is complete demixing $\sigma^{2}{ }_{0}$. The masses of the three components are nearly the same.

In all experiments, the rotating speed is varied in the range of $10-40 \mathrm{rpm}$. The sample mass for sieving is set to 12 samples of

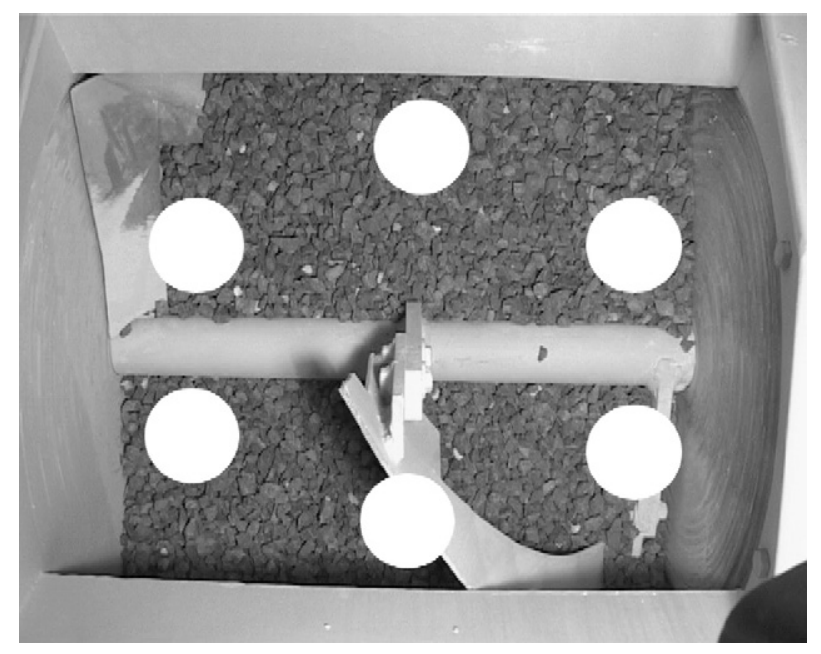

Fig. 7. Sampling points.

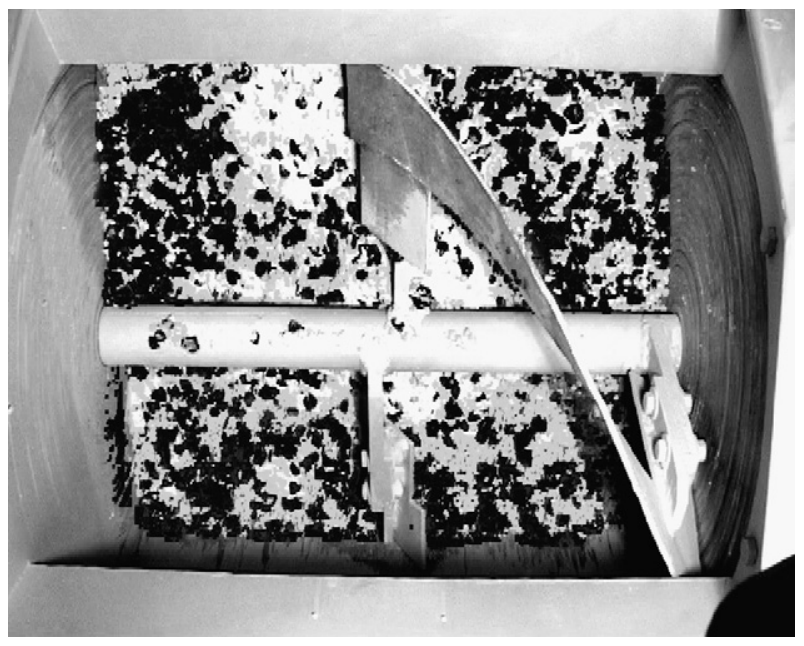

Fig. 8. Distribution after $t_{M}=40 \mathrm{~s}$ mixing time.

$400-600 \mathrm{~g}$ each. The mixing quality is determined at $20 \mathrm{~s}, 40 \mathrm{~s}$, $60 \mathrm{~s}$, and $80 \mathrm{~s}$. Samples are taken at the points specified in the mixing volume. These points are located at maximum distances from each other, such that adjacent sampling points are not affected by sampling. The sampling points are shown in Fig. 7. The mixing times for determining the mixing quality by image analysis range between $0-120 \mathrm{~s}$. The camera will take photos in an interval between fixed times with an exposure time of $250 \mathrm{~ms}$. Fig. 8 shows the mixing state after $40 \mathrm{~s}$ mixing time at a rotating speed of $10 \mathrm{rpm}$. Demixing of the coarse material can be observed at the edges of the cylindrical housing in particular. The fine component is highly enriched at certain points of the mixer. At other points of the mixer, better mixing is achieved.

For image analysis, the Image J 1.34 S software of the Wayne Rasband National Institutes of Health USA (http://rsb.info.nih. gov/ij/) is used. For the analysis of the individual pixels, this program only detects the color black or white. The resolution of the digital images is $1024 \times 768$ pixels. The separated digital images from the Adobe Photoshop in Figs. 9 and 10 are colored black, orange and white after separation. The image J transforms the different particle fractions into a binary image (black or white) to analyze the pixels. From the counted pixels, the total surface area of the individual particle fractions is obtained.

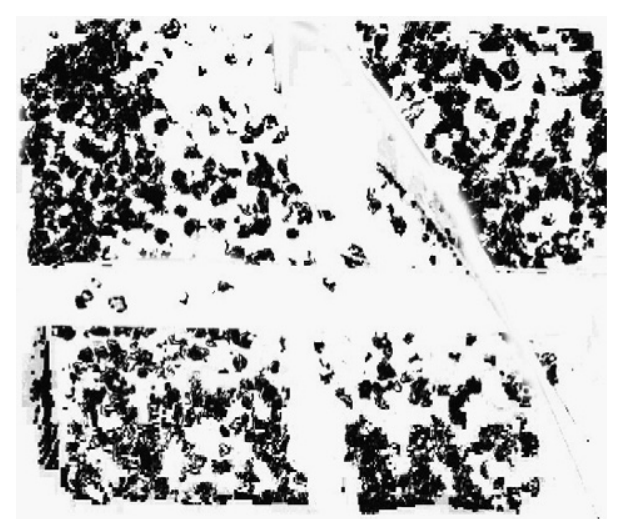

Fig. 9. Distribution of the coarse fraction. 


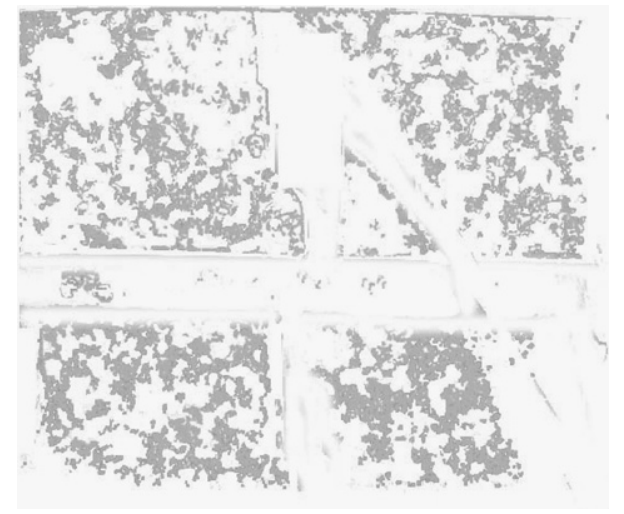

Fig. 10. Distribution of the medium-sized fraction.

By means of Image $J$, individual particle surfaces can be analyzed. For this type of surface analysis, particles of the same color must not be in contact with each other. This type of analysis is impossible, see Fig. 9. When plotting the surface distribution $Q_{2}$ of the individual areas versus the number of individual particle surfaces according to Fig. 11, it is found that most of the surface fraction does not exist as an individual particle. For a homogeneous surface distribution of the individual particles, a straight line would be obtained according to Fig. 10. This optimum mixing cannot be achieved, as demixing prevents an optimum distribution at the surface. A statistical analysis according to Eq. (2) yields variation coefficients $v$ of $200-300 \%$ for the individual surfaces.

This method cannot be applied to determine the local particle distribution for the mixing of dry components. Further analysis focuses on the total surface area at a certain mixing time. The results are shown in Fig. 12 for two rotating speeds. The normalized total surface fraction of the components $\mathrm{j}$ is plotted versus the number of revolutions $\Theta$. In this way, the results obtained at various rotating speeds can be compared.

It is found that the demixed initial state has no influence on the respective surface distribution. It is also shown by the results that the coarse fraction is enriched at the surface with about $50 \%$ of the total surface area. The medium-sized and the fine fractions are in the range of $21-26 \%$, i.e. they are below the target value of $33 \%$. The finer components trickle through the moved bed earlier than the coarse fraction.

Using the equations given in Section 2, the mixing qualities result as a function of the number of revolutions $\Theta$. The mixing qualities are plotted in Figs. 13, 14 and 15 for the coarse fraction, the medium-sized fraction, and the fine fraction, respectively. The mixing qualities obtained from sieving $\sigma_{S}^{2}$ and image analysis $\sigma_{B}^{2}$ reveal that the variation coefficients in the stationary end state are nearly identical (see Fig. 12). The stationary equilibrium is reached at about $\Theta=10$ revolutions in both image analysis and sieving. The mixing quality $\sigma_{B}^{2}$ by image analysis is determined from twelve mixing experiments. The sample for the image analysis is the total surface of the single-shaft mixer what has approximately $A_{\text {ges. }}=0.09 \mathrm{~m}^{2}$. By comparing the two methods sieving and image analysis to determine the mixing efficiency the individual sample mass of $m_{P} \approx 600 \mathrm{~g}$ has to be constant.

The mixing efficiency of the medium-sized component shown in Fig. 13 exhibits a behavior that is similar to that of the coarse component. A difference can be observed for the unstationary mixing process. At revolutions smaller than $\Theta=10$, both mixing qualities do not have the same coefficients. During the start-up procedure for the medium-sized fraction, differences occur between the surface and the bulk. This results in various variation coefficients. The mixing efficiencies obtained for the fine component are presented in Fig. 14. The mixedness versus time curve obtained from sieving lies above the image analysis curve. Both curves are nearly parallel, in particular at the beginning of mixing. The difference between both curves may be explained by the measurement error during sampling from the mixing volume. The sampling unit, here, a shovel, must be designed for the sample mass and the compact bulk. In case of fine materials, this type of sampling unit inevitably results in a non-defined material loss that causes the measurement error. The stationary final state of both

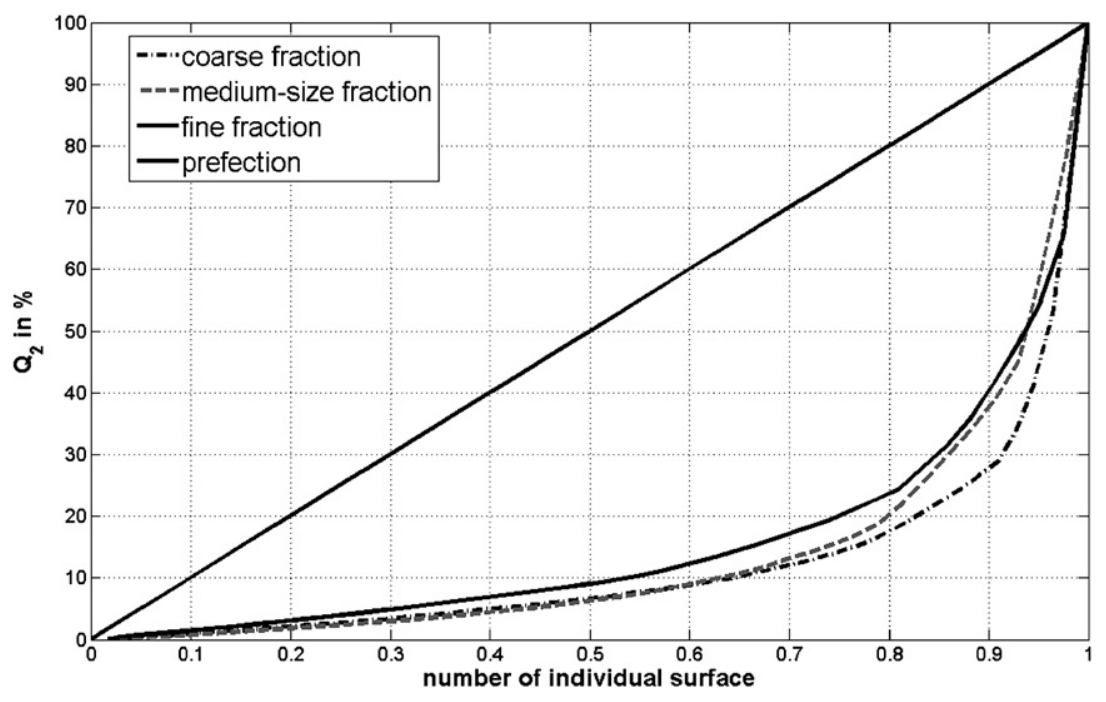

Fig. 11. Surface distribution versus the number. 


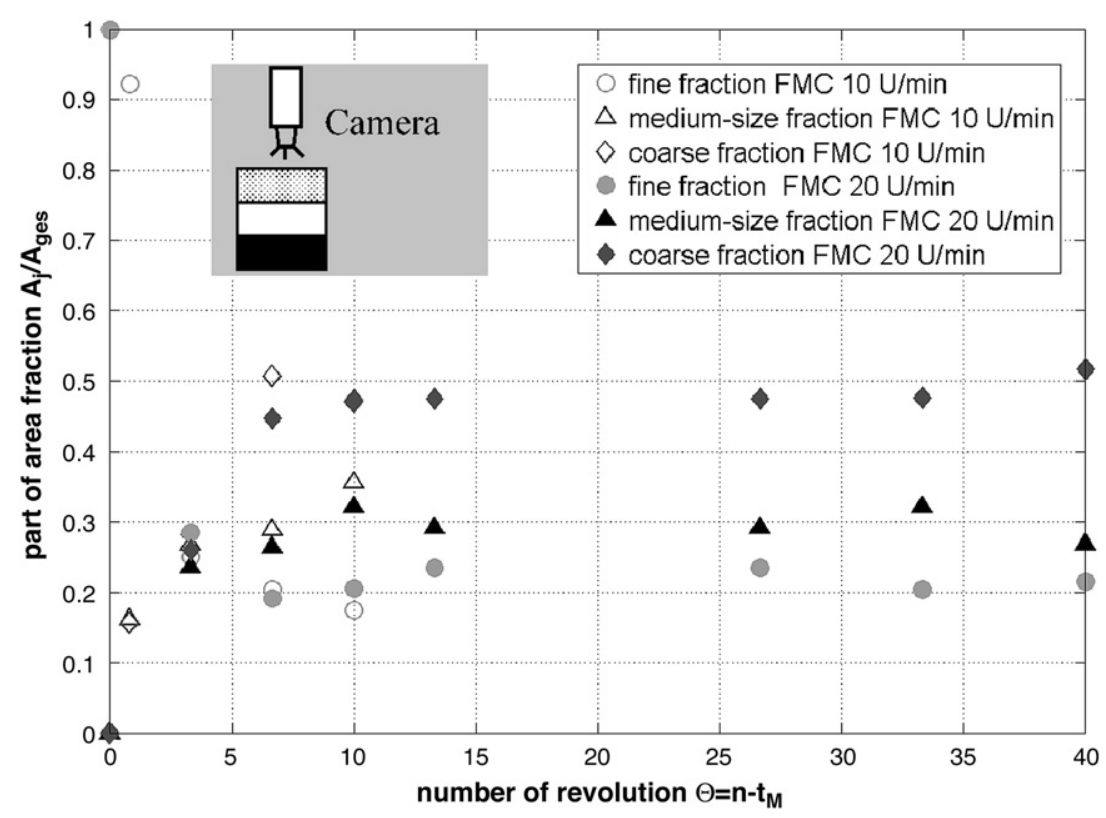

Fig. 12. Surfaces of the three fractions with time of individual surfaces.

curves is reached at $\Theta=10$ revolutions. In all of this mixing efficiency figures the stationary equilibrium shows a small oscillation. The reason for this is the motion of the mixing paddles during the mixing process. Sometimes there is more or less surface to analyze, due the rotation and shadow of the mixing paddles.

The results are confirmed by comparisons with practical studies of Beitzel and Charonnat [6]. With this type of solid mixtures, stationary homogeneity occurs after mixing times of
$t_{M}=30-50 \mathrm{~s}$. At rotating speeds of $20-25 \mathrm{rpm}$, this corresponds to $10-20$ revolutions.

The experiments doesn't show the mixing quality of the whole solid mixer, because the separation is a kinetic effect from the surface to the ground. Authors like Hong [7] and Brone [8] wrote about the effect by segregating different particle sizes. In Daumann [9] the separation will be reduced if liquid will be added to the bulk materials.

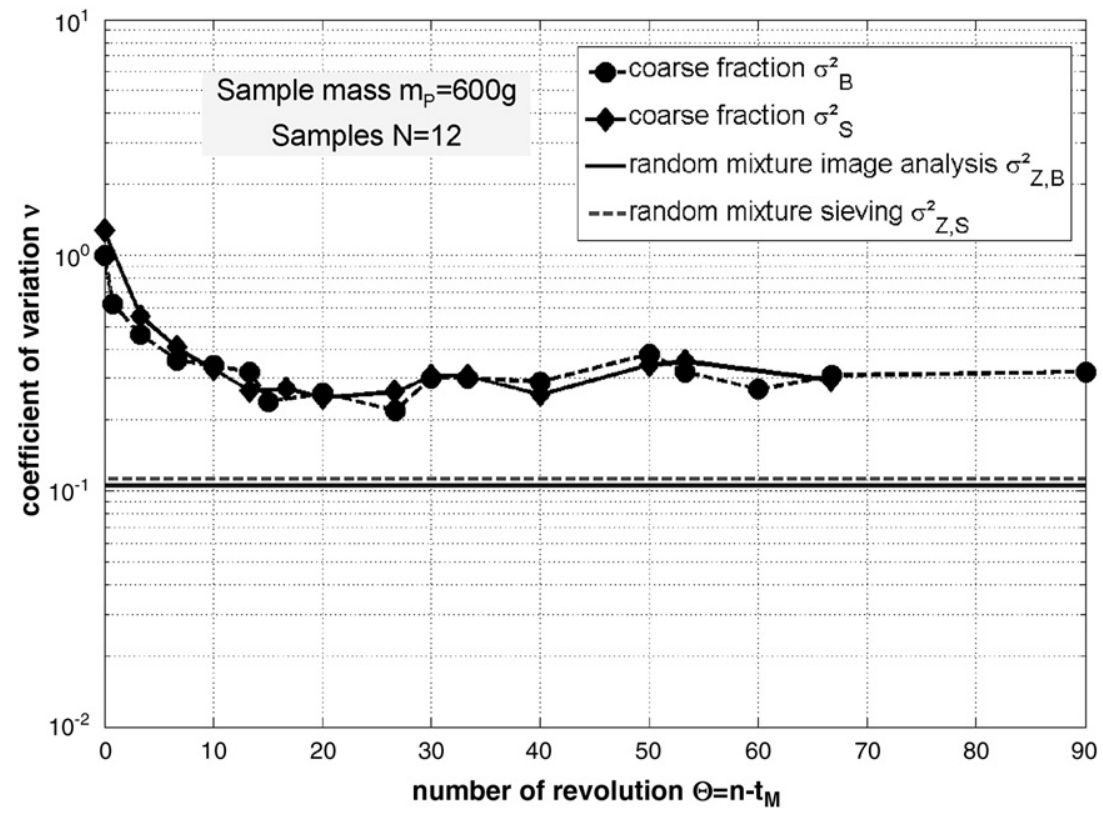

Fig. 13. Mixing efficiency of the coarse fraction versus the number of revolutions. 


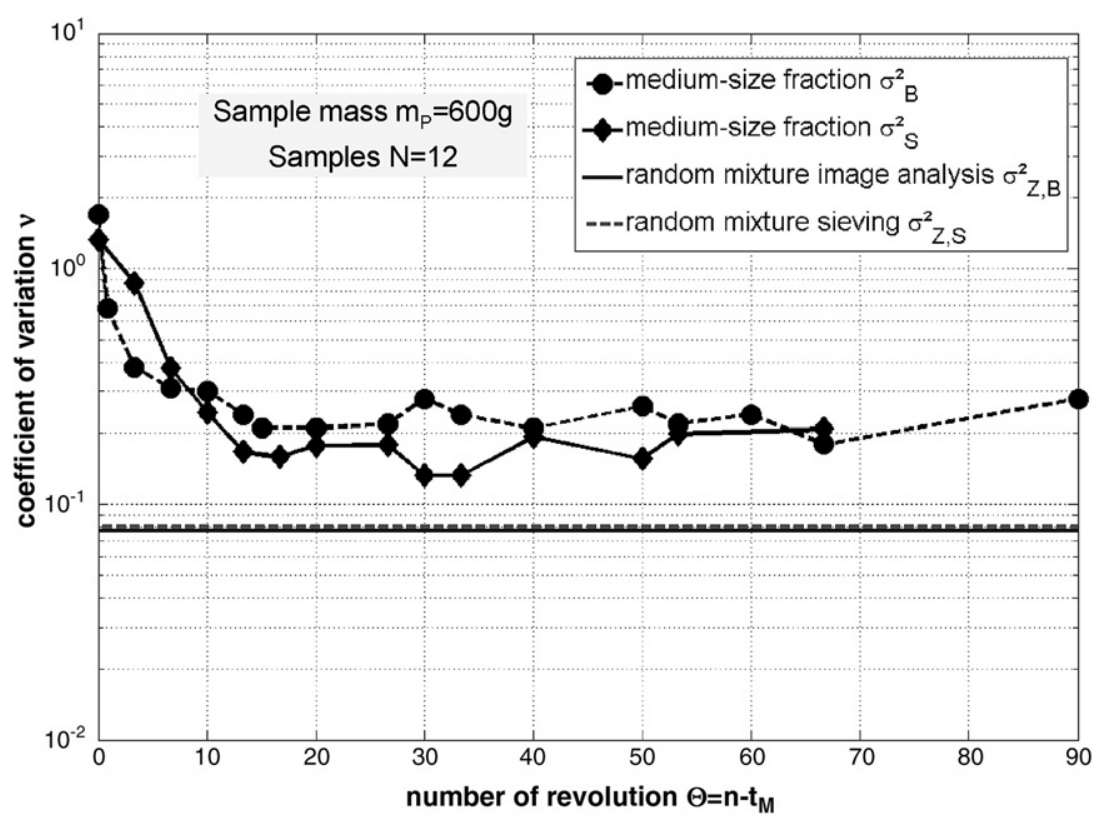

Fig. 14. Mixing efficiency of the medium-sized component versus the number of revolutions.

The reason why the two mixing efficiencies are very close to each other is, that taking a sample of mass from the bottom of the bulk material is impossible, because the structure of the mixture is a compact mineral material which doesn't allow further sampling from a deeper position. The depth of the sample of mass has a maximum of $15 \mathrm{~cm}$.

\section{Summary}

Image analysis allows to describe the mixing efficiency at the surface of the mixing volume. It is found that in case of dry mixtures, it is impossible to determine the local distribution of individual particles at a mixing time $t_{M}$, since large ranges of

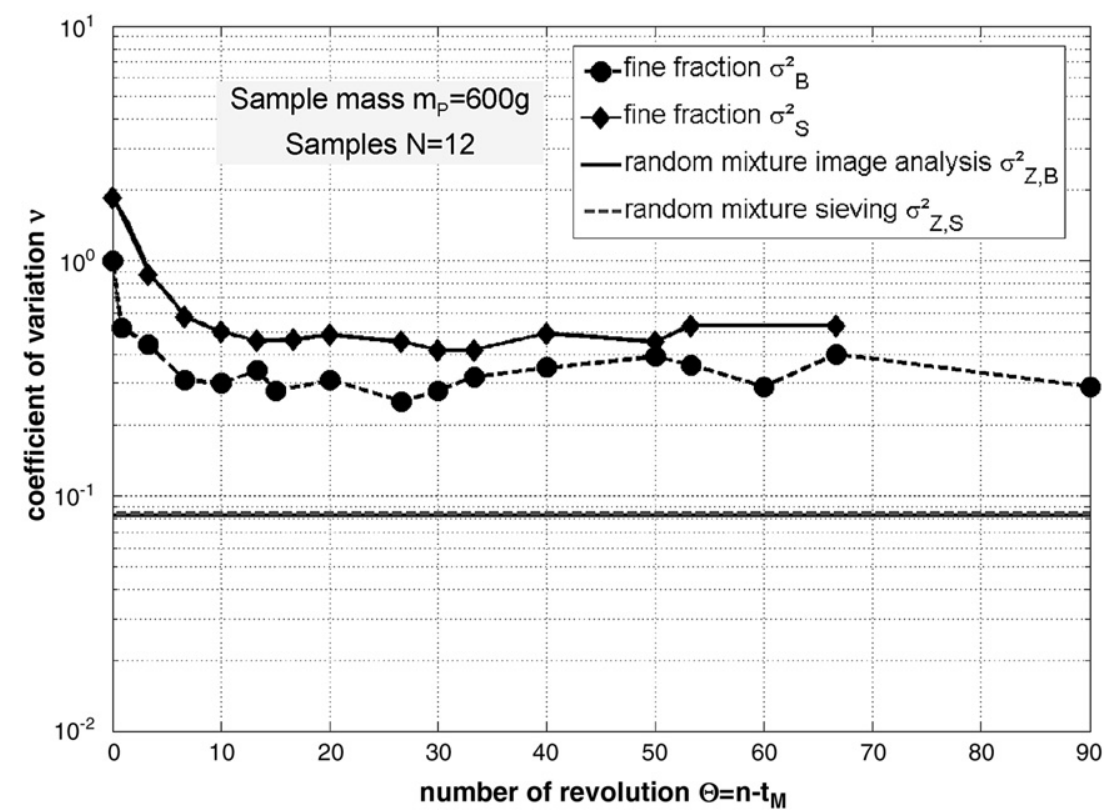

Fig. 15. Mixing efficiency of the fine component versus the number of revolutions. 
particles of identical color are in contact with each other. The image analysis program considers this coherent surface to be one particle. Due to various single particle areas, surface demixing does not allow for a statistical analysis at the surface. A variance $\sigma_{B}^{2}\left(X_{j}\right)$ of the surface fractions at different mixing times yields nearly the same variances as a distribution of the concentration in the bulk. The method of image analysis can be applied to describe the mixing efficiency and to obtain the optimum point of stationary equilibrium. Having reached this equilibrium, no further improvement of the mixing quality is achieved in spite of the further movement of the mixing tool. This method allows statements to be made with respect to the mixing behavior of solids within a relatively short term and without time-consuming sampling and sample analysis. The method is not able to represent the mixing behavior of the whole mixture, because the segregation on the surface, caused by the different particle sizes, avoid this. But the method of image analysis can be used to mark one component to examine the mixing behavior.

\section{Symbols}

$A \quad$ Surface area of the mixing volume $\left[\mathrm{m}^{2}\right]$

$A_{\text {ges }} \quad$ whole surface area of the mixer

$D \quad$ Diameter of the mixing tank [m]

$D_{\mathrm{W}} \quad$ Diameter of the mixing equipment [m]

j Fraction

$m_{P} \quad$ Sample mass [kg]

$L \quad$ Length of the mixing tank [m]

$m_{E, j} \quad$ Mass of the individual grain [kg]

$N \quad$ Number of samples

$n \quad$ Rotating speed $\left[\mathrm{s}^{-1}\right]$

$N_{E, j} \quad$ Number of individual particles [-]

$P \quad$ Target concentration $[-]$

$P_{E} \quad$ Expected value [-]

$P_{K} \quad$ Mass fraction of the component $k[-]$

$Q_{2} \quad$ Surface distribution [\%]

$Q_{3} \quad$ Volume distribution [\%]

$s^{2} \quad$ Empirical variance [-]

$t_{M} \quad$ Mixing time $[\mathrm{s}]$

$t_{S} \quad$ Student factor [-]

$X \quad$ Measured concentration [-]

$x_{\mathrm{pm}} \quad$ Mean projected diameter $[\mathrm{m}]$
$x_{R} \quad$ Residual humidity [\%]

$x_{S} \quad$ Equal surface diameter [m]

$x_{V} \quad$ Equal volume diameter [m]

$\Theta \quad$ Number of revolutions [-]

$v \quad$ Variation coefficient [-]

$\rho_{S} \quad$ Solid density $\left[\mathrm{kg} / \mathrm{m}^{3}\right]$

$\rho_{\text {bed }} \quad$ Bulk density $\left[\mathrm{kg} / \mathrm{m}^{3}\right]$

$\sigma_{\mathrm{B}}^{2} \quad$ Variance of image analysis [-]

$\sigma_{\mathrm{M}}^{2} \quad$ Variance of the measurement value [-]

$\sigma_{\mathrm{S}}^{2} \quad$ Variance of sieving [-]

$\sigma_{\mathrm{Z}}^{2} \quad$ Variance of the random mixture [-]

$\psi \quad$ Sphericity $[-]$

\section{Acknowledgements}

The authors acknowledge the financial support granted by the Federal Ministry of Economics and Technology (BMWi). In addition, the authors would like to thank Elba GmbH, Germany for the supply of the laboratory-scale single-shaft mixer.

\section{References}

[1] K. Sommer, G. Hauser, Determination of mixing times of a paddle mixer with rapid discharge for production of dry mortar and plaster, Aufbereitungstechnik/Mineral Processing 6 (1989) 367-374.

[2] J. Raasch, B. Elsässer, Compare the mixing quality between a continuous solid mixer and discontinuous solid mixer (only German language available), Schüttgut 2 (3) (1995) 497-503.

[3] K. Sommer, Sampling of Powders and Bulk Materials, Springer Verlag Berlin Heidelberg New York, Tokyo 1986 ISBN 0-387-15891-X (U.S.).

[4] W. Alex, Principles and systematics of the counting procedure for particle size analysis (only German language available), Aufbereitungstechnik/ Mineral Processing 2 (1972) 105-111.

[5] H. Wadell, Sphericity and roundness of rock particles, The Journal of Geology 41 (1933) 310.

[6] H. Beitzel, Y. Charonnat, Efficiency of concrete mixers towards qualification of mixers, Materials and Structures Rilem (1997) 28-32.

[7] D.C. Hong, P.V. Quinn, S. Luding, Reverse Brazil nut problem: competition between percolation and condensation, Physical Review Letters 86 (15) (2001) 3423-3426.

[8] D. Brone, F.J. Muzzio, Size segregation in vibrated granular systems: a reversible process, Physical Review Letters E 56 (1997) 1059-1063.

[9] B. Daumann, H. Nirschl, Influence of the mixing system on dry and moist bulk solids, Aufbereitungstechnik/Mineral Processing 48 (4) (2007) 19-33. 